OAI-PMH: http://www.indteca.com/ojs/index.php/Revista Scientific/oai

Artículo Original / Original Article

\title{
Teoría de acción humana en las organizaciones: Una visión desde la gestión humanista
}

Autor: Michael Josué Aular Galindo Universidad Nacional Experimental Simón Rodríguez, UNESR mikeaular@hotmail.com Caracas, Venezuela https://orcid.org/0000-0003-1248-2484

\section{Resumen}

El presente artículo tiene como objetivo establecer la congruencia entre la Teoría Explícita y la Teoría en Uso, de los gerentes con respecto a una gestión humanista y de esta manera dejar espacio a las acciones pertinentes que ayuden a posicionar al ser humano en el centro de la gestión organizacional. La investigación tiene un enfoque cuantitativo, de tipo descriptiva, en la que participaron cuatro gerentes y veinte colaboradores directos, se llevó a cabo en Caracas y el estado Miranda, Venezuela. Tras aplicar el instrumento de recolección de datos (cuestionarios), se obtuvieron resultados que indican un alto nivel de incongruencia entre lo que declaran 0 dicen hacer y lo que practican o realmente hacen los gerentes con relación a una gestión humanista. Estos resultados contribuyeron a resaltar la importancia de crear conciencia en este tema, abrir espacios de reflexión sobre la Teoría en Uso y así explorar la naturaleza del ajuste necesario para el cambio de paradigma en la gestión organizacional.

Palabras clave: acción humana; gestión humanista; gerencia; teoría de acción.

Cómo citar este artículo:

Aular, M. (2021). Teoría de acción humana en las organizaciones: Una visión desde la gestión humanista. Revista Scientific, 6(19), 228-242, e-ISSN: 2542-2987. Recuperado de: https://doi.org/10.29394/Scientific.issn.2542-2987.2021.6.19.11.228-242

Fecha de Recepción: 14-09-2020
Fecha de Aceptación: 10-12-2020
Fecha de Publicación: 05-02-2021 
OAI-PMH: http://www.indteca.com/ojs/index.php/Revista Scientific/oai

Artículo Original / Original Article

\title{
Theory of human action in organizations: A vision from humanistic management
}

\begin{abstract}
The objective of this article is to establish the congruence between the Explicit Theory and the Theory in Use, of the managers with respect to a humanistic management and in this way leave space for the pertinent actions that help to position the human being at the center of the organizational management. The research has a quantitative approach, of a descriptive type, in which four managers and twenty direct collaborators participated, it was carried out in Caracas and the state of Miranda, Venezuela. After applying the data collection instrument (questionnaires), results were obtained that indicate a high level of incongruity between what they declare or say they do and what managers practice or actually do in relation to humanistic management. These results contributed to highlighting the importance of raising awareness on this issue, opening spaces for reflection on the Theory in Use and thus exploring the nature of the adjustment necessary for the paradigm shift in organizational management.
\end{abstract}

Keywords: human action; humanistic management; management; theory of action.

\footnotetext{
How to cite this article:

Aular, M. (2021). Theory of human action in organizations: A vision from humanistic management. Revista Scientific, 6(19), 228-242, e-ISSN: 2542-2987. Recovered from: https://doi.org/10.29394/Scientific.issn.2542-2987.2021.6.19.11.228-242
}

Date Received: 14-09-2020
Date Acceptance:

10-12-2020
Date Publication: 05-02-2021 


\section{Introducción}

En la dinámica organizacional de hoy, muchas son las dificultades con las que deben lidiar los líderes conjuntamente con sus colaboradores, entre las que se encuentran el cómo mejorar las relaciones laborales de forma armoniosa y productiva, como también lo referente a contar con acciones que ayuden a construir una gestión donde las personas se sientan más identificadas, desarrolladas, valoradas, respetadas y tomadas en cuenta, todo esto teniendo presente la necesidad de contar con un equilibrio entre la importancia de la productividad como también la construcción del bienestar de los integrantes de la organización.

En este orden de ideas, una de las principales funciones del gerente es fortalecer la organización y las relaciones humana, el lograr que otras personas hagan algo muchas veces se convierte en un reto diario y es que para regir las acciones humanas hacen falta condiciones precisas que impulsen ese transitar hacia los objetivos propuestos, muchas veces se observa que los gerentes dicen estar de acuerdo con la aplicación de un estilo de gestión de carácter humanista, pero en la práctica no muestran compromiso con todo lo que implica esta manera de gestionar, creando así incongruencias entre lo que dicen hacer o estar de acuerdo y lo que realmente hacen.

En ese sentido, la finalidad de este tipo de acciones (humanistas), es lograr que los integrantes de las organizaciones como un sistema; generen compromiso, alto nivel de participación en las decisiones, identidad, responsabilidad, gratitud, colaboración y excelencia; sean capaces de aprender permanentemente y desarrollen redes de comunicación efectivas a todo nivel de la entidad y así contribuir al bienestar colectivo.

De igual forma, como gerentes comprometidos con esta manera de gestionar deben apoyar la creación de espacios en el que todos los colaboradores se sientan cómodos participando en las decisiones importantes de la organización, que se consideren escuchados y tomados en cuenta, 
aunado a esto, Rodríguez (2020), afirma que:

Las decisiones para que sean importantes deben tomarse en equipo, así los trabajadores asumen los éxitos y fracasos de la empresa y a tener responsabilidades en la complejidad de las decisiones gerenciales a enriquecer las responsabilidades en las oportunidades de crecimiento, compartir con los trabajadores y no hacerlo todo, dejar que ellos opinen y tomen algunas decisiones, les da oportunidades para enseñar lo que han aprendido por experiencia en la organización [...] (pág. 170).

Con base a la situación antes descrita, este trabajo se plantea el objetivo de establecer la congruencia entre la Teoría Explícita y la Teoría en Uso de los gerentes con respecto a una gestión humanista y así dejar espacio a las acciones pertinentes que ayuden a lograr esta manera de gestionar las organizaciones, generando un aprendizaje en ese trayecto. Con relación al aprendizaje, Argyris (1999a): considera que se produce [...] cuando detectamos y corregimos un error. Un error es cualquier discordancia entre lo que queremos que produzca una acción y lo que sucede en realidad cuando implementamos esa acción. Es una discordancia entre las intenciones y los resultados" (pág. 19). A continuación, se delinean las principales teorías, que se utilizaron en la presente investigación como guía y sustento.

\subsection{Teoría de Acción Humana de Argyris y Schön}

Desde la perspectiva de Argyris y Schön (1978a): la acción humana no es casual, sino con intención, cuando se actúa se hace con un fin en sí, el ser humano construye un tipo de conocimiento (práctico, tácito) para orientar sus acciones, además evalúa sus consecuencias y hace ajustes de ser necesario, todo esto permite estudiar los aprendizajes organizacionales.

De hecho, Argyris y Schön (1978b): explican que "los individuos responden al error modificando estrategias y supuestos dentro de las normas organizativas constantemente" (pág. 37); reestructurando de esta forma su 
teoría de acción. En este sentido, el comportamiento de cualquier persona está asentada en una teoría de acción que él mismo ha erigido para orientarse en el entorno existente.

Aunado a esto como teoría, en gran medida es una vía de control o normativa y sirve para explicar y predecir lo que es la acción del individuo u organización, estos autores investigaron el comportamiento de las personas y grupos, los mapas mentales que los sujetos construyen para operar en diversas situaciones, como también los aspectos en la cultura organizacional que en gran medida bloquean el actuar acorde al objetivo de generar el aprendizaje. En efecto, Argyris y Schön (1978): refieren que "cuando atribuimos teorías de la acción a los seres humanos, argumentamos que toda acción deliberada tenía una base cognitiva, que reflejaba normas, estrategias y supuestos o modelos del mundo que tenían pretensiones a la validez general" (pág. 10).

La teoría de acción de cualquier sujeto se presenta en dos dimensiones, la Teoría de Acción Explícita: es el conjunto de creencias valores y normas que cada persona tiene de forma declarada. En esta dimensión se identifica lo que una persona expresa con respecto a lo que tiene para actuar de determinada forma ante una situación y la Teoría de Acción en Uso: es el conjunto de creencias valores y normas que gobiernan el comportamiento real de la persona, es decir la que aplica habitualmente. Con relación a esto, Sulbarán (2001), manifiesta que:

Existe desde el punto de vista individual una teoría que se predica, conformada por los fines, supuestos y valores que, de acuerdo al individuo, guían su conducta; y una teoría que se practica, configurada por los supuestos implícitos que en realidad guían la conducta observada [...] (pág. 8).

Estas dimensiones pueden ser opuestas entre sí y la persona muchas veces es inconsciente de ello, como expresa Argyris (1999b): "a menudo hay 
discordancias fundamentales, sistemáticas entre los diseños que los individuos defienden y los que emplean" (pág. 79). Esto resulta útil saberlo, ya que de esta forma se podría ayudar a configurar los principios de acción, las normas, supuestos, entre otros componentes de la teoría de acción del sujeto; con la finalidad de lograr un mayor grado de eficiencia.

\subsection{Gestión Humanista}

Desde las primeras teorías y prácticas de la administración (revolución industrial) está presente el ser humano, bien sea con un papel simplista, mecanicista, reduccionista, pero de una u otra forma ha sido parte de la misma, a través del tiempo lo que ha venido evolucionando es el grado de importancia que se le da al ser humano en el mundo organizacional. Vinculado a esto, González (2007a): enfatiza que "[...] no se debió a una intencionalidad contraria al ser humano, sino a que partieron de premisas falsas acerca de la naturaleza humana, que en su momento histórico se daban por ciertas" (pág. 43).

En este orden de ideas, el respeto por la dignidad humana se hace más formal, cuando entre otros aspectos se visualiza y expone al ser humano que hace vida en las organizaciones desde un enfoque más integral, resaltando aspectos como la motivación que nace de las necesidades sociales y personales de los trabajadores, las potencialidades del ser humano, el valor limitado del dinero para la satisfacción, la tendencia de demagogia, la asignación de responsabilidades, la integración. Con respecto a lo antes planteado, se realiza énfasis en las consecuencias de no tomar en cuenta la naturaleza humana en la dinámica empresarial como también el uso del saber para la época de las ciencias sociales en el plano organizacional. Con relación a esto, González (2007b), plantea que precisamente:

Basado en las teorías de Abraham Maslow, fundador de la Psicología Humanista, McGregor proponía una nueva forma de 
mirar al ser humano en el contexto del trabajo industrial. Modificó el concepto taylorista del hombre de la organización y presentó un nuevo paradigma que hace énfasis en el potencial humano [...] (pág. 46).

Aquí se da inicio también a esa meta de demostrar que se puede optar por mejorar las condiciones de trabajo para las personas y garantizar la productividad de las organizaciones. Es así como a finales del siglo XX, una gran cantidad de académicos e investigadores, se interesan en desarrollar aún más esta corriente de las ciencias administrativas. A inicios del siglo XXI se forman asociaciones para investigar y discutir sobre el humanismo en la gestión de las organizaciones, hoy día unas de las más conocidas y de trayectoria son, la Red de Gestión Humanística con sede principal en Suiza, pero con capítulos en una gran cantidad de países, la escuela de Montreal y el Centro de Humanismos, Gestión y Mundialización Latinoamérica.

Ahora bien, Melé (2003): considera que la gestión humanista es aquella que "enfatiza en la condición humana y está orientada al desarrollo de las virtudes humanas, en todas sus formas, en toda su extensión" (pág. 79). Por consiguiente, ante esta manera de concebir al ser humano en el trabajo, es indispensable aceptar sus valores, creencias, emociones objetivas, necesidades y potencialidades, así como también la importancia real que éste tiene en los diversos procesos de la organización.

En este sentido es pertinente resaltar que, la gestión humanista surge como una alternativa para resolver los desequilibrios producidos por el paradigma económico tradicional, basado en el Homo œconomicus, en el cual se modeliza el comportamiento humano y su valor fundamental es el de la utilidad. Bajo esta nueva perspectiva se plantea, posicionar al ser humano al centro de la gestión de cualquier organización, en palabras de Chanlat (2003): "[...] las preocupaciones humanistas deberán ser plenamente integradas en las prácticas cotidianas de la gestión y dejar de ser vistas como piezas 
añadidas o simples votos piadosos" (pág. 52).

Por consiguiente, una organización que respeta, promueve y protege la dignidad humana, práctica la medición y disminución de los impactos negativos en la sociedad que opera, estimula las capacidades de cada miembro del equipo, apoya que cada colaborador asuma de forma autónoma su rol y así comprenda el papel esencial que tiene sus funciones, acepta al ser humano como un ser complejo por naturaleza, busca el bienestar de todos poniendo como centro a las personas, brinda un ambiente laboral agradable y propicio a la productividad, entiende el desarrollo económico compartido como un medio para lograr estabilidad y justicia; se puede decir qué es una entidad con una gestión humanista.

Desde esta perspectiva, García, Portales y Arandia (2014), citados por Arandia y Portales (2015): afirman que "la razón de ser de una organización constituida bajo este modelo de gestión debe tomar como primer punto el desarrollo del ser humano" (pág. 128). Es así que conocer la realidad de la problemática relacionada a la congruencia entre la Teoría Explícita y la Teoría en Uso de los gerentes con respecto al desarrollo de esta manera de gestionar reviste una gran relevancia, ya que a nivel nacional e internacional es un tema que demanda conciencia, esfuerzos y compromiso para el cambio de un paradigma donde el ser humano es un medio para un fin económico, a uno donde el desarrollo, bienestar y dignidad del ser humano es el fin de toda actividad humana.

\section{Metodología}

El presente trabajo se desarrolló desde una perspectiva cuantitativa a través de la conformación de una muestra total de 24 sujetos, integrado por 4 gerentes y 20 colaboradores directos, en el cual se planteó establecer la congruencia entre la Teoría explícita y la Teoría en Uso de cada gerente con respecto a la gestión humanista. 
En cuanto al diseño y muestra, se realizó un estudio de tipo descriptivo, de diseño no experimental. Con relación al estudio descriptivo Hernández, Cuevas, Méndez y Mendoza (2017): destaca que por medio de este "se busca especificar las propiedades, características y perfiles de personas, grupos, comunidades, procesos, objetos o cualquier otro fenómeno que se someta a un análisis" (pág. 76). La muestra estuvo compuesta por veinticuatro (24) personas en total (13) mujeres y (11) hombres, constituida por cuatro (04) gerentes de organizaciones venezolanas, con y sin fines de lucro, uno por cada organización y veinte (20) colaboradores directos, (5) por cada gerente, los cuales debían tener laborando al menos un año en esa gerencia, las edades oscilaban entre los 23 años hasta los 58 años, la técnica de muestreo fue la no probabilístico, por conveniencia.

Se utilizó la encuesta como técnica y el cuestionario como instrumento, consta de 16 interrogantes, validado mediante la técnica de juicio de expertos con una escala de respuesta tipo Likert y probada su confiabilidad mediante el coeficiente Alfa de Cronbach, generando 0,96 de confiabilidad (alta). Este estudio integra además un análisis comparativo de los resultados.

Finalmente, en cuanto al proceso metodológico, luego de una revisión bibliográfica para identificar las características propias de una gestión humanista, se diseñaron y aplicaron dos tipos de cuestionarios. El primero dirigido a gerentes de cuatro organizaciones aplicado durante el tercer trimestre del año 2020, para determinar la Teoría Explícita de cada uno de ellos y un segundo cuestionario a los colaboradores directos de estos gerentes, con la finalidad de identificar la Teoría en Uso de estos y así poder establecer la congruencia o no entre ellas.

Con respecto al cuestionario, es propio del método cuantitativo, Hernández, Ramos, Placencia, Indacochea, Quimis y Moreno (2018), declara que:

Proporciona información sobre un mayor número de personas 
en un período de tiempo más breve, que se cuantifica, analiza e interpreta con facilidad. La finalidad del cuestionario es obtener, de forma sistemática y ordenada, la información de la población investigada sobre el objeto de investigación (pág. 118).

Se destaca la importancia de revelar que los participantes en el presente estudio, acordaron un consentimiento de uso de información y de igual forma, la confidencialidad de los nombres de las personas y de las organizaciones participantes.

\section{Resultados}

La presente investigación ha identificado los principales hallazgos con respecto al establecimiento de la congruencia entre la Teoría Explícita (lo que dicen hacer) y la Teoría en Uso (lo que realmente hacen) los gerentes estudiados.

Es posible identificar en el tema de llevar a cabo acciones recurrentes para conocer, respetar y tomar en cuenta las opiniones de los colaboradores, el $75 \%$ de los gerentes dice estar muy de acuerdo mientras otro $25 \%$ expresa estar de acuerdo, al contrastar estas respuestas con lo que realmente hacen, conforme a las contestaciones recolectadas de sus colaboradores, existe una incongruencia de un $75 \%$ entre lo que dicen y lo que hacen, solamente en un caso los colaboradores confirmaron la Teoría Explícita del gerente.

En cuanto al estudio, en necesario contar con acciones que les demuestre empatía, reciprocidad, solidaridad y confianza a los colaboradores, evidenciándose que en la totalidad de los gerentes, contestaron estar muy de acuerdo, al contrastar estas respuestas con lo que realmente hacen y, acorde con las contestaciones recolectadas de sus colaboradores, existe una incongruencia de un $75 \%$ entre lo que dicen y lo que hacen, solamente en un caso, los colaboradores confirmaron la Teoría Explícita del gerente. 
Con relación a tener acciones que aseguren un crecimiento económico compartido como, por ejemplo, sistemas de remuneración justos, seguridad social, planes de reconocimiento entre otros beneficios a los colaboradores, la mitad de los gerentes respondieron estar muy de acuerdo y el restante respondieron estar de acuerdo. Al contrastar estas respuestas con lo que realmente hacen de acuerdo con las consultas recolectadas a sus colaboradores, la totalidad de estos dijeron estar totalmente en desacuerdo.

En el caso de ofrecer y apoyar planes de formación profesional de forma constante a los colaboradores, el $75 \%$ de los gerentes indicaron estar muy de acuerdo mientras otro $25 \%$ señaló estar de acuerdo, en la práctica según respuestas recolectadas de sus colaboradores no tienen acciones que demuestren dicho apoyo con un margen del 75\%, que respondieron estar en total desacuerdo.

Otro tema que se estudió fue lo concerniente al apoyo de la generación de espacios formales e informales de dialogo que propicien una comunicación constante y efectiva entre todos los integrantes del equipo de trabajo. Los gerentes respondieron en su totalidad estar totalmente de acuerdo con este hecho. Por su parte las respuestas obtenidas de los colaboradores en un $100 \%$ fue estar totalmente en desacuerdo con la existencia de estas acciones por parte de la gerencia.

También se indagó, sobre sí la gerencia apoya y practica el empoderamiento de cada colaborador. Resultando que el $75 \%$ de los gerentes están totalmente de acuerdo con aplicar este tipo de acciones. Según las respuestas recolectadas el $100 \%$ de los colaboradores están en desacuerdo con la existencia de este tipo de acciones por parte de la gerencia.

Para culminar, como se observa en el gráfico 1, de los 4 gerentes que participaron en este estudio, solamente 1 tiene un alto nivel de congruencia (con un $94 \%$ de confirmación) entre su Teoría Explícita y su Teoría en Uso, lo que representa un $25 \%$ del total de la muestra, según el análisis general de 
Artículo Original / Original Article

los datos recogidos.

Gráfico 1. Gerentes con congruencia entre su Teoría Explícita y su Teoría en Uso.

\section{CONGRUENCIA ENTRE} TEORÍAS

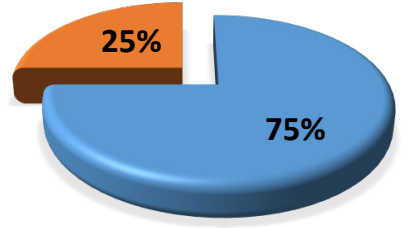

Fuente: El Autor (2020).

\section{Conclusiones}

Los resultados de la investigación muestran, que existe un alto grado de incongruencia entre lo que dicen hacer y apoyar los gerentes y lo que realmente hacen con respecto a una gestión humanista, si bien en las ciencias administrativas se viene haciendo un gran esfuerzo para el apoyo de esta forma de gestionar las organizaciones, aún no es ampliamente aplicada.

En este sentido, a pesar de que se ha logrado gradualmente la aceptación de la importancia de lo humano en las organizaciones, resulta preocupante la falta de acciones concretas por parte de los gerentes para migrar efectivamente del modelo donde el ser humano es un medio para el logro de un fin económico, a uno en el que es el centro de toda actividad.

Es así como, con la presente investigación se recomienda seguir sumando esfuerzos para propiciar aprendizajes y conciencia en este tema, abrir espacios de reflexión sobre la Teoría en Uso con relación a una gestión humanista y de esta forma explorar la naturaleza del ajuste necesario para el 
cambio de paradigma en la gestión organizacional. Este hecho es algo que debería analizarse en profundidad desde las aulas de clases de los programas de administración y gerencia, porque es un proceso en el cual está inmerso hondamente la creación de sensibilización en los líderes sobre el impacto de contar con esta manera de gestionar hoy día.

\section{Referencias}

Arandia, O. \& Portales, L. (2015). Fundamentos de la gestión humanista: una perspectiva filosófica. AD-minister, (26), 123-147, e-ISSN: 22564322. Recuperado de: https://doi.org/10.17230/ad-minister.26.6

Argyris, C. (1999a,b). Conocimiento para la Acción: Una Guia Para Superar los obstáculos del Cambio en la Organización. ISBN: 84-7577-6434. Barcelona, España: Ediciones Juan Granica.

Argyris, C., \& Schön, D. (1978). Organizational Learning: A Theory of Action Perspective. 1st Edition, ISBN: 0-201-00174-8. United States: Addison Wesley Publishing.

Chanlat, A. (2003). Gestiones y Humanismos: Una Arqueología de la gestión. Iztapalapa: Revista de Ciencias Sociales y Humanidades, (55), 35-52, e-ISSN: 0185-4259. Recuperado de:

https://dialnet.unirioja.es/servlet/articulo?codigo=6092345

González, L. (2007a,b). Humanismo y Gestión Humana: Una Perspectiva de Interpretación para el Trabajo Social Aplicado al Campo Laboral. Revista Eleuthera, 1, 42-63, ISSN: 2011-4532. Colombia: Universidad De Caldas - Unicaldas.

Hernández, A., Ramos, M., Placencia, B., Indacochea, B., Quimis, A. \& Moreno, L. (2018). Metodología de la investigación científica. Primera Edición, ISBN: 978-84-948257-0-5. Alcoy, España: Área de Innovación y Desarrollo, S.L.

Hernández, R., Cuevas, A., Méndez, S., \& Mendoza, C. (2017). Fundamentos 
de Investigación. ISBN: 978-607-15-1395-3. México: McGraw-Hill / Interamericana.

Melé, D. (2003). The Challenge of Humanistic Management. Journal of Business Ethics, 44, 77-88, e-ISSN: 1573-0697. Recovered from: https://doi.org/10.1023/A:1023298710412

Rodríguez, M. (2020). Aproximación Teórica de la Concepción del Gerente Agrícola: Una Visión Transcompleja (Zona del Páramo, Mérida). Revista Scientific, 5(16), 162-178., e-ISSN: 2542-2987. Recuperado de: https://doi.org/10.29394/Scientific.issn.2542-2987.2020.5.16.8.162-178 Sulbarán, J. (2001). La Teoría de Acción: Posibilidades de Aplicación en el Ámbito Universitario. Actualidad Contable Faces, 4(4), 7-16, eISSN: 1316-8533. Recuperado de:

https://www.redalyc.org/articulo.oa?id=25700402 


\section{Artículo Original / Original Article}

\section{Michael Josué Aular Galindo \\ e-mail: mikeaular@hotmail.com}

Nacido en Caracas, Venezuela, el 3 de marzo del año

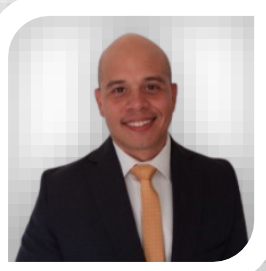
1987. Doctorando en Ciencias Administrativas en la Universidad Nacional Experimental Simón Rodríguez (UNESR); Especialista en Gerencia empresarial en la Universidad Santa María (USM); Máster en Gerencia Estratégica Empresarial en el Centro de la Organización de las Naciones Unidas para la Educación, la Ciencia y la Cultura (UNESCO); Licenciado en Contaduría Pública en la Universidad Alejandro de Humboldt (UAH); actualmente me desempeño como docente de la escuela de Contaduría Pública en la Universidad Alejandro de Humboldt; y Facilitador en el Instituto de Desarrollo Profesional de los Colegios de Contadores Públicos del Distrito Capital y el Estado Miranda (IDEPROCOP); así como consultor empresarial.

El contenido de este manuscrito se difunde bajo una Licencia de Creative Commons ReconocimientoNoComercial-Compartirlgual 4.0 Internacional 